\title{
La figura de "la Sombra" en el espacio doméstico: sobre el arquetipo de la Sombra en cuentos fantásticos escritos por mujeres a finales del siglo XIX
}

\author{
Natacha ANDRADA DE GREGORIO \\ Universidad Complutense de Madrid \\ natacha.andrada@gmail.com
}

Recibido: 15.09 .2012

Aceptado: 30.09 .2012

\begin{abstract}
RESUMEN
Este artículo se centra en cómo el arquetipo de la Sombra creado por C. G. Jung está representado en los cuentos escritos por mujeres pertenecientes al llamado gótico femenino. De forma consciente o inconsciente las mujeres literatas de una época determinada (finales siglo XIX) utilizaron este arquetipo junguiano, en sus diferentes facetas, para deshacerse de sus represiones y temores, proyectándolo en sus espacios domésticos. La Sombra se convierte en el reflejo de la frustración y del dolor. Así, las mujeres comenzaron su proceso de individuación con sus relatos fantásticos tal y como queda reflejado en los cuentos que se analizan: El empapelado amarillo (1892) de Charlotte Perkins Gilman y La mujer lobo (1890) de Clemence Housman.
\end{abstract}

Palabras clave: arquetipo, Sombra, gótico femenino, proceso de individuación, frustración.

\section{The Shadow Archetype and Domestic Space in Women's Short Stories of the Late Nineteenth Century}

\begin{abstract}
This essay analyzes how the Shadow Archetype in Jungian Psychology is represented in the women's literature belonging to the female gothic. Consciously or unconsciously, the literary women of a specific period (the end of the nineteenth and beginning of the twentieth Century) used this Jungian archetype, in its different phases, to dissolve their repressions and fears. The Shadow Aspect becomes a projection of their sorrow and frustration. Women start their individuation process with their ghost tales, as it is shown in the stories analyzed here: The Yellow Wallpaper (1892) by Charlotte Perkins Gilman, and Werewolf (1890) by Clemence Housman.
\end{abstract}

Keywords: archetype, shadow, Female gothic, individuation process, female frustration.

"Hoy hacia el anochecer 
me adentré un poco con la niña ciega

en el bosque donde todo es sombra y oscuridad.

La acompañé hacia una sombra que venía a nuestro encuentro.

Le acarició las mejillas con sus dedos de terciopelo

y ahora a ella también

le gustan las sombras.

Y el miedo que tenía se ha ido"1.

Opal Witheley.

En la sociedad patriarcal que dominaba en el siglo XIX, la literatura ayudó a las mujeres a expresar su identidad y a vencer la moral impuesta durante siglos. Uno de los mecanismos más explorados de las escritoras para enfrentarse al concepto de lo que los hombres entendían por femenino fue la transformación de los personajes femeninos en sus relatos. Para llevar a cabo la creación de protagonistas transgresoras, muchas de las escritoras recurrieron al género gótico. En este género las autoras encontraron el amparo literario que no podían hallar en otros y les servía, además, para criticar lo que la sociedad esperaba de ellas. Numerosos estudios desde una perspectiva feminista demuestran cómo en el género gótico escrito por mujeres se refleja la abundante represión y frustración que sufrían las autoras de la época. Por otro lado, C. G. Jung nos ha demostrado muy a menudo que el relato de las fantasías contiene siempre, no sólo los problemas más perentorios del narrador, sino lo que por el momento es lo más doloroso de sus problemas íntimos (Jung, 2005: 59). De esta forma, el género gótico se convertiría también en un mecanismo catártico para las escritoras.

Teniendo en cuenta esta visión del género gótico escrito por mujeres como un género que esconde lo que la mujer reprime, y aplicando las herramientas conceptuales que C. G. Jung nos da para la exploración del inconsciente, este estudio analiza el arquetipo de la Sombra en dos relatos representativos del gótico femenino del siglo XIX. Dado que el gótico fue un mecanismo de liberación para las autoras, éstas llegan a identificarse con las protagonistas que ellas crean, reflejando en sus relatos sus frustraciones e instintos negados. Así, el estudio refleja cómo la Sombra de las mujeres víctimas de la sociedad patriarcal no está formada por lo que ellas quieren ocultar inconscientemente (por sentimientos de vergüenza o de culpa) sino que está formada por las represiones que la sociedad patriarcal impone. Al descubrir cuáles

\footnotetext{
${ }^{1}$ Poema citado en Mujeres que corren con los lobos (Estés, 1998).
} 
son los secretos ocultos en su Sombra, se llegará a la conclusión de que a través de la utilización de este arquetipo, las autoras expresan con plena libertad los deseos reprimidos que comparten tanto las protagonistas de sus cuentos como ellas mismas.

\section{INTRODUCCIÓN A LA SOMBRA}

El descubrimiento del inconsciente por Freud y las teorías que a partir de él se han desarrollado han supuesto un avance decisivo en el estudio del aspecto psicológico de las obras de arte. Su relación con literatura es innegable. Por otro lado, C. G. Jung, discípulo del primero, exploró el inconsciente colectivo y descubrió técnicas metodológicas para la elaboración de sus teorías. Entre estas técnicas metodológicas está la elaboración de imágenes del inconsciente que fueron llamadas por Jung «arquetipos». Los arquetipos pertenecen a la memoria colectiva del ser humano, existen en todas las civilizaciones, en sus mitologías y en su cultura. El término debe entenderse como un principio organizador que nos ayuda a comprender la realidad. En sus palabras los arquetipos son el oculto tesoro que la humanidad ha acumulado y del que ha producido sus dioses y sus demonios, y todos aquellos pensamientos poderosos e influyentes sin los cuales las personas dejarían de ser humanas (Jung, 2007: 64). En otras palabras, son estructuras formales dotadas de autonomía y espontaneidad que se manifiestan y se canalizan a través de la imaginación creadora y de las funciones del Yo: éste puede fascinarse y aterrarse con los arquetipos o incluso ser dominado y poseído por ellos.

Aún de forma esquemática es preciso señalar cómo Jung concibe la organización y el funcionamiento de la personalidad humana ${ }^{2}$. En dicha personalidad ocupan el lugar central dos factores estructurales: el Sí-mismo (Selbst) y el Yo (Ich). El Selbst es el punto central de la totalidad de lo psíquico y comprende la extensión de la conciencia y del inconsciente, así como el Ich es el centro de la conciencia (Jung, 1957: 57). Así pues, el Selbst es el eje central de la personalidad total, lo que yo verdaderamente soy como sujeto humano, y el Ich ocupa el lugar central respecto a la conciencia y al nivel biográfico. El Ich está situado como mediador entre el universo interno personal y arquetípico, y el mundo externo físico y sociocultural (Vázquez, 2001: 154). Ahora bien, este eje formado por el Selbst y el Ich se complementa con otros tres factores estructurales fundamentales: la Persona, la Sombra y el Alma (Anima/Animus). El concepto de «Persona» representa el arquetipo de la máscara, simboliza la cara exterior del sujeto en sociedad, lo que aparenta ser, no lo que es. La «Sombra» es el

\footnotetext{
${ }^{2}$ Para un conocimiento más a fondo sobre este tema recomiendo acudir al libro Psicología de la personalidad en C. G. Jung (VÁQUEZ, 1981).
} 
lado oscuro de la personalidad pero no sólo lo negativo de cada uno, también lo reprimido, lo negado y lo temido tanto a nivel individual como colectivo. El término de «Alma» comprende prácticamente todo lo psíquico o anímico $\mathrm{y}$, en otras ocasiones, significa sólo la personalidad interior en contraposición a la «Persona» (Vázquez, 2001: 156). En los varones, el Anima, corresponde a la imagen femenina ideal que proyectan en la mujer. Del mismo modo, el Animus se encuentra en las mujeres y corresponde a la imagen masculina proyectada en el varón.

Tras un breve y sintético recorrido por la estructuración de la personalidad según C. G. Jung, es conveniente precisar cómo el psiquiatra expresa la auténtica realización del sujeto humano. C. G. Jung propone un recorrido para llegar a ser individuos completos; un recorrido que el alma humana realiza de manera natural aunque su duración es diferente en cada individuo. Este recorrido es llamado el proceso de individuación que consiste en la asimilación y actualización de las figuras arquetípicas. Teóricamente, deberán integrarse en una secuencia: en primer lugar el sujeto desenmascara su «Yo», es decir, deja de identificarse con la «Persona», deja de vivir la apariencia y se despoja de sus máscaras frente a la sociedad. En segundo lugar, reconoce e integra su «Sombra», se acepta tal como es. En tercer lugar, explora su «Alma», proceso formado por una doble tarea: comienza a cultivar su vida interior, en el campo psíquico y espiritual, y retira sus proyecciones anímicas del otro sexo. Por último, el sujeto se deja iluminar por el arquetipo del sentido espiritual de la vida (personificado en los mitos por el viejo sabio) hasta que se encuentra finalmente con «sí-mismo», con el fin último del proceso: el Selbst, el cual representa al sujeto individuado (indivisible) en una integridad psíquica.

No obstante, el proceso más relevante para este estudio es el relativo al enfrentamiento con la «Sombra» y por ello exige una mayor explicación. Ésta, pues, es el segundo arquetipo al que se debe hacer frente en el camino de la individuación y está a su vez formada por dos niveles de energía psíquica: uno es lo proveniente del inconsciente colectivo y otro lo proveniente del inconsciente personal. Lo proveniente del inconsciente colectivo en este arquetipo contiene los elementos tabú de la humanidad que han permanecido en la «Sombra» a través del tiempo (se pueden encontrar referencias al parricidio, al incesto o a la antropofagia, entre otras). Este inconsciente colectivo nos remonta al hombre primitivo y animal guiado por los instintos primarios. Por otro lado, los componentes personales de la «Sombra» se forman con el tiempo y están más vinculados con la historia de cada vida. Son todos aquellos elementos no apreciados por la consciencia (aquello que nos hiere o nos humilla, entre otros) y determinados por la educación y la cultura. Así, la «Sombra» está conformada por contenidos que no hemos desarrollado y por elementos que negamos de nosotros mismos. 
En el camino de la individualización hemos de pasar por situaciones en las que se niega la «Sombra», se proyecta $\mathrm{y}$, finalmente, se interioriza. De esta forma el individuo se conciencia de la propia «Sombra» y consigue una aceptación integral de sí mismo. Las palabras del psicólogo Pedro Daniel González García explican de forma concreta y comprensible dicho camino:

"Al observar la Sombra y animarse a dar ese primer paso en busca de los secretos que se esconden en las penumbras, estaremos iniciando el camino que nos lleve a la comprensión de que luz y obscuridad no son mas que dos extremos de una misma cosa, y que sin la permanencia de uno, el otro no podría continuar existiendo. (...) Se manifestará lo que ocurre en nuestro interior, y una vez comprendido esto, se podrá iniciar un dialogo con la propia Sombra, que, a su vez, dará la pauta para buscar ese equilibrio que nos llevará a ser más honestos y coherentes con nosotros mismos y nos ayudará retornar a aquello que verdaderamente somos, un individuo completo" (González García, 2009).

¿Cómo manifestamos este arquetipo de la «Sombra»? De nuevo, las palabras de los estudios de psicología analítica sirven de ayuda para una mejor comprensión:

"Puede ser a través de sueños, fantasías o proyecciones, pero siempre será del mismo sexo que el sujeto, incluso al ser proyectada. Cuando es vivenciada por medio de sueños y fantasías, tomará su apariencia dependiendo de lo cerca o lejos que se encuentre dentro del plano de la consciencia, mientras más negada se tenga, se manifestará en símbolos más alejados de la persona, tomando formas no humanas, que podrían ser animales, y gradualmente se irá aproximando, pudiendo tomar formas subhumanas como figuras monstruosas humanoides, después tomaría formas humanas pero alejadas de la realidad del sujeto, como gente de otras etnias o primitivos, hasta llegar a figurar como algún conocido. Realizando el recorrido desde lo desconocido y temido hasta llegar a lo mas cercano" (González García, 2009).

De este modo, todo lo que el sujeto niega, reprime, oculta o rechaza de su personalidad, lo proyecta en los otros, consiguiendo una falsa sensación de liberación de su lado oscuro. Sin embargo, de forma gradual, el sujeto ha de aceptar su realidad, desprenderse de la angustia de la culpabilidad e integrar en sí mismo su propia «Sombra» para su realización completa.

\section{LA REPRESENTACIÓN DE LA SOMBRA EN LA LITERATURA GÓTICA FEMENINA}

Los cuentos seleccionados para este estudio fueron escritos por mujeres en la sociedad victoriana durante la segunda mitad del siglo XIX: una época en la que lo femenino se identificaba con lo inferior o marginal, las mujeres aún no participaban 
prácticamente en ninguna actividad política o cultural y mantenían una relación conflictiva entre su vida pública y sus inquietudes privadas. Conviene señalar al respecto que el icono de mujer como ángel del hogar, que surgió a partir de un poema de Coventry Patmore (1823-96) en el que idealizaba a su mujer en su rol doméstico como compañera sumisa, entregada y abnegada y celebrando el amor matrimonial, fue el más extendido en la sociedad del siglo XIX. En la mujer se buscaba castidad, sumisión y obediencia al marido, modestia, pureza y abnegación como virtudes fundamentales. Este prototipo femenino no solamente fue promovido por Patmore ni por filósofos como Jean Jacques Rosseau en su obra El Contrato Social y Emilio o de la educación sino que también la ciencia y la medicina contribuyeron a la formación de esta mujer casta y asexual. Como explica Anna Clark en su obra Deseo. Una historia de la sexualidad en Europa:

"El conocimiento del cuerpo femenino también varió a lo largo de los siglos. Durante los inicios de la era moderna, por ejemplo, muchos manuales médicos y populares sobre sexo describían el clítoris como el foco del placer en las mujeres y el orgasmo femenino como necesario para la concepción (...). A finales del siglo XVIII y principios del XIX, tal como demostró Thomas Laqueur, los médicos descubrieron que el orgasmo femenino no era necesario para la concepción y el clítoris desapareció de sus textos" (Clark, 2010: 18).

La burguesía inglesa se hallaba en los momentos de mayor vigor y unos claros valores conservadores (que afectaban sobre todo a la mujer) dominaban la sociedad. Si la mujer debía ser ahorradora, discreta, sumisa, dócil, dispuesta, asexual y moralmente pura, sus impulsos más fuertes y primitivos, así como sus necesidades intelectuales, estaban siendo negados, rechazados y escondidos y, por lo tanto, dejados en sombra. Por ello, desde mediados del siglo XIX, surgió en Inglaterra un renovado auge de los relatos de fantasmas escritos por mujeres que combinaban de forma magistral los elementos sobrenaturales con los elementos cotidianos y servía para las autoras como una forma literaria autorizada a través de la cual podían criticar, rebelarse y reaccionar contra el modelo femenino que predominaba en las obras de los hombres. La creación comenzaba a traspasar el ámbito masculino. Este resurgido género gótico se llenó de textos donde plasmaron sus miedos, frustraciones y deseos ocultos; textos que se convirtieron en el espejo de sus pensamientos; textos donde lo reprimido retornaba.

De esta forma, el género gótico escrito por la mujer victoriana ha sido caldo de cultivo para aportar a su lectura una perspectiva feminista, representada en el término female gothic (gótico femenino) tal como lo utiliza y explica Ellen Moers, en una de sus obras más influyentes, Literary Women: 
"What I mean by Female Gothic is easily defined: the work that women writers have done in the literary mode that, since the eighteenth century we have called the Gothic" (Moers, 1978: 85).

Críticas feministas han hecho hincapié en que las literatas utilizaban un lenguaje ambiguo, de doble dirección, y una amplia gama de tácticas para oscurecer pero no borrar sus impulsos más subversivos (Gilbert \& Gubar, 1979: 20). Patricia Meyer Spacks señala repetidas veces que las obras de las mujeres escritoras ocultan pensamientos subterráneos incluso a veces escondidos a ellas mismas (Gilbert \& Gubar, 1979: 89). Annis V. Pratt, por su parte, encuentra en estas obras toda una serie de amagos, tramas, máscaras y disfraces insertados (Gilbert \& Gubar, 1979: 90). Las investigaciones de Elaine Showalter, Ellen Moers, y Gilbert y Gubar que propusieron nuevas vías para acercarnos a la lectura de las novelas escritas por mujeres, indican que las literatas del siglo XIX tuvieron una subcultura literaria femenina y claramente definida (GilberT \& Gubar, 1979:12). Lo que busca la escritora es su identidad desde un punto de vista propio y no el que ha gestado el hombre a lo largo de la historia, buscan su imagen no estereotipada. A través de sus personajes redefinen sus vidas, atacan y revisan su biografía, se construyen, escriben a partir de símbolos que habitan en lo más profundo de la mente, símbolos que parten de la soledad o de la furia, de la frustración e incertidumbre, o del miedo y la angustia. Sus mentes crean monstruos y vampiros, fantasmas de niños muertos, locas recluidas, aristócratas diabólicos, mujeres lobo, heroínas malditas y perseguidas, o ángeles del hogar rebeldes bajo la apariencia de la femineidad permitida.

Por ello, en este florecer literario, puede encontrarse el arquetipo de la Sombra no sólo como representación del lado oscuro, sino también como reflejo de lo reprimido y escondido. Mediante el enfrentamiento con la Sombra, las protagonistas de los cuentos encontrarán una imagen más completa y libre de ellas mismas. Este reflejo de la «Sombra» se llevará a cabo en espacios aterradores. En ocasiones se tratará de espacios cercanos a ellas mismas que se transforman de forma siniestra. El terreno familiar e íntimo que, en un principio, se construye para brindar seguridad y refugio a la mujer, se transmuta en algo desconocido, inquietante e, incluso, aterrador; lo extraño se apoderará de lo doméstico produciendo una sensación de profunda angustia.

\subsection{EL EMPAPELADO AMARILLO (1892) DE CHARLOTTE PERKINS GILMAN.}

Este conocido relato de la autora estadounidense Charlotte Perkins Gilman (18601935) esconde mucho más de lo que aparenta en una primera lectura. Una lectura superficial nos enfrenta con un relato de terror: una mujer enferma cree discernir una mujer atrapada bajo el empapelado amarillo que cubre su habitación y enloquece rasgando el papel de la pared, para poder liberarla. Desde esta perspectiva se trataría 
de un cuento de fantasmas y apariciones en el que lo inanimado cobra vida y el miedo está presente. Entonces, el empapelado amarillo tendría algún tipo de cualidad demoníaca y sobrenatural. Sin embargo, la interpretación psicológica y feminista es más compleja y va mucho más allá del mero relato gótico: el texto podría representar el ascenso a la libertad; un verdadero manifiesto contra el sometimiento de la mujer en la época victoriana.

Este relato se ha leído también como un escrito semi-autobiográfico. La autora fue paciente del Doctor Weir Mitchell, especialista en el sistema nervioso, quien aplicaba a sus pacientes una terapia que combinaba reposo en cama, aislamiento, sobrealimentación y aplicación de descargas eléctricas de una manera estrictamente regulada. A Charlotte Perkins Gilman, que sufrió lo que hoy entendemos como "depresión postparto", se le diagnosticó neurastenia, se le separó de su bebé y, como cura, se le prohibió leer y escribir o realizar cualquier tipo de trabajo intelectual. Pues bien, la protagonista del relato sufre la misma condena: está obligada a estar en cama y sólo a escondidas consigue escribir para, como ella dice, liberarse. Gilman también utilizó la escritura como mecanismo catártico: cuando logró superar su enfermedad, pese a que la "cura" podría haberla llevado a la locura, decidió escribir su relato para dar una lección sobre los supuestos remedios médicos (peores que las enfermedades que pretenden remediar). Una muestra más de su fortaleza la encontramos al final de su vida: con 72 años le diagnosticaron un cáncer de pecho y ella decidió que no iba a ceder ante la enfermedad. Compró una botella de cloroformo y la guardó para cuando su vida dejase de ser útil. Así, el 17 de agosto de 1935 realizó su se suicidó y dejó una carta que aparece en su autobiografía póstuma. La carta acababa declarando:

"Cuando toda la utilidad se ha extinguido, cuando la muerte es segura, inevitable e inminente, elegir una forma rápida y fácil de morir, en lugar de una lenta y horrible, es el más básico de los derechos humanos (...). Al creer que esta elección es un servicio social para que pueda promover unos puntos de vista más sabios sobre este tema, he preferido el cloroformo al cáncer" (Ceplair, 1991: 276).

Es importante señalar la importancia del espacio donde tendrán lugar las representaciones del arquetipo de la Sombra. El espacio familiar no es cómodo y agradable para la protagonista, es extraño e inquietante. Desde el primer momento el lector se envuelve en ese ambiente de misterio que encierra la casa alquilada donde la narradora y su esposo pasarán el verano: “¿Por qué será de alquiler tan barato esta casa? ¿Y por qué llevará tanto tiempo sin alquilar? (...) hay algo extraño en esta casa, algo que puedo sentir claramente" (Gilman, 1892: 131). Lo mismo sucederá con los objetos personales. La cama, lugar propicio para el descanso y la comodidad, le produce sensaciones de agobio y aislamiento: "Estoy tumbada en esta gran cama inamovible que se me antoja clavada al suelo-, siguiendo el empapelado durante horas. Puedo dar fe de que hacerlo es tan bueno como la gimnasia" (Gilman, 1892: 138). 
Desde una interpretación junguiana, el arquetipo de la Sombra encajaría en la mujer empapelada que hay tras el papel amarillo; ese papel que desde un principio disgusta tanto a nuestra protagonista: "Es como si dos ojos bulbosos, sobre un cuello roto, salieran de una parte donde el dibujo se repite hasta la saciedad y te mirasen al revés" (Gilman, 1892: 136). Poco a poco la protagonista asume su frustración, lo reprimido y lo temido se acercan a la consciencia, y el papel va adquiriendo forma y cara; comienza a convertirse en algo animado y vivo: "Cuando le da la luz del sol de lleno, puedo observar una suerte de figura extraña, incluso provocadora e informe" (Gilman, 1892: 137). A medida que la Sombra se asimila, se va definiendo su figura: "Bajo el empapelado crece día a día una forma oscura. Es siempre la misma forma única, aunque parezca multiplicada. Es como una mujer que reptara encorvada por la parte más baja de la pared, bajo el dibujo del papel amarillo" (Gilman, 1892: 141). Finalmente, cuando el sujeto se enfrenta de forma completa a su Sombra y se asume en la personalidad, se produce la síntesis total de la mujer protagonista con la mujer empapelada:

"Ya no quiero ni mirar por la ventana. Hay demasiadas mujeres arrastrándose por ahí a gran velocidad. Me pregunto si todas ellas, como yo, habrán salido del empapelado amarillo de la pared (...) en cuanto se me haga de noche me veré obligada a esconderme otra vez tras ese espantoso dibujo del empapelado" (Gilman, 1892: 149).

Si analizamos el cuento como una experiencia de la autora convertida en literatura, la protagonista sería su doble, su alter ego. A su vez, la Sombra de la protagonista es la mujer empapelada. Por lo tanto, la mujer empapelada es la Sombra de la autora. Ambas proyectan en su sombra su represión, sus ocultas ansias de libertad. La mujer empapelada representa su lado escondido, su asfixia en un mundo en el que su palabra no es ni válida ni suficiente para decidir por sí misma. Un mundo en el que le ha sido impuesto un tratamiento de reposo y por el que llega a enloquecer. La mujer empapelada y silenciada es esa faceta oculta que clama por salir a la superficie y ser escuchada. Es la mujer que quiere rasgar su opresión, que se siente encarcelada y no lo acepta. La protagonista, en cambio, es la faceta de la mujer exterior, la que no pude dejarse dominar por sus pasiones y ha de cumplir con lo que la sociedad espera de ella. Siguiendo la teoría de Jung, el proceso de asimilación de la Sombra supondría una aceptación de la parte oculta, herramienta que ayuda en la transformación de individuos completos. Como si de una metáfora de este proceso se tratase, la escena final muestra como la aceptación de la sombra devuelve a la protagonista una imagen más libre de ella misma. Pese a que ha sido necesaria la locura, consigue pasar por encima de aquello que le niega la libertad:

"Yo seguí arrastrándome por el suelo, igual que antes, pero le miraba alzando la cabeza. Al fin he conseguido salir de ahí - dije -, a pesar de ti y de Jennie... Y como he arrancado la mayor parte del papel, ya no podrás volver a confinarme contra la 
pared ¿Por qué se desmayaría este hombre? Porque se desmayó, cayendo junto a la pared. Y para seguir arrastrándome tuve que pasar por encima de él" (Gilman, 1892: 150)

Finalmente, una mención a la crítica conseguida por Gilman contra el sistema del momento y la presión y nulidad a la que estás sometidas las mujeres. Gilman denuncia el papel que se espera de ellas y su falta de autonomía. La mujer se halla empapelada en la imaginación y en la realidad, en el cuento y en la vida. La autora allana el camino a las mujeres posteriores animándonos implícitamente a no callar y a manifestar el descontento para no acabar "empapeladas".

\subsection{LA MUJER LOBO (1890) DE CLEMENCE HOUSMAN}

El hombre lobo forma parte de nuestro imaginario desde tiempos ancestrales y ha ocupado desde textos literarios hasta antropológicos, religiosos y filosóficos, periodísticos y, por supuesto, mitos y leyendas. Ya encontramos textos grecolatinos con hombres lobo, como el rey Licomedes o el tirano Licaón, del que se dice dio origen al nombre de licantropía. La licantropía, tal y como cuenta el escritor Jorge Fondebrider en su estudio sobre las historias de hombres lobo en Occidente, tuvo un nuevo auge literario en el siglo XIX en Inglaterra. Nos aclara que el relato más antiguo es de procedencia alemana, aunque sólo se conoce su posterior traducción inglesa. Se trata de un cuento escrito por Johann Apel en la primera década del siglo, que hacia 1840 alcanzó gran éxito en Inglaterra bajo el título de The Boar Wolf. En 1833 la revista The Story-Teller había publicado otro sin firma, The Wehr-Wolf, manifiestamente escrito años después que el anterior. Sin embargo, el primer gran clásico del género es The White Wolf of the Hartz Mountains, publicado en 1837 por el inglés Frederick Marryat (1792-1848) (Fonderbrider, 136). Numerosos autores del siglo XIX escribieron historias sobre hombre y mujeres lobo: Charles de Coster (Werewolf, 1867), Robert Louis Stevenson (Olalla, 1887), Sir Gilbert Campbell (White Wolf of Kostopchin, 1889), Rudyard Kipling (The Mark of the Beast, 1889), entre otros.

Pese a estar menos explorada, la mujer lobo encarna el arquetipo de la Sombra proveniente del inconsciente colectivo: el lado oscuro femenino que pone de manifiesto el poder seductor y sexual de la mujer, un plano temido y atractivo al mismo tiempo. Aquí, la Sombra, hace referencia a esas características animales, entre las que se encuentra la sexualidad, que la sociedad patriarcal ha intentado adormecer.

Como ejemplo literario de este personaje se encuentra el texto referido: La mujer lobo, de Clemence Housman. Montague Summers, importante crítico de literatura fantástica en la primera mitad del siglo XX, declaró el relato de Housman un exquisito poema en prosa narrado con un sentimiento tan poco común como hermoso 
(Navarro en Housman, 1890: 242). Clemence Housman (1861-1955) fue una importante feminista que apoyó incondicionalmente el voto para las mujeres y fundó diversas instituciones para la ayuda a la mujer entre las que destaca el Suffrage Atelier, fundada junto con su hermano Laurence Hosuman y descrita como An Arts and Crafts Society Working for the Enfranchisement of Women. Llevó a cabo una intensísima actividad política sin detenerse hasta que se aprobó el sufragio universal, combinándola a la perfección con su faceta artística. Su relato La mujer lobo, una mujer fuerte y luchadora, representa bien sus ideales. Fue publicado por entregas en la revista inglesa Atalanta entre 1890 y 1891, y más tarde publicada en un volumen único por Lane, Way \& Williams Publishers en 1896. El éxito fue rotundo y fue reeditada en numerosas ocasiones.

La historia narra como dos hermanos gemelos, Sweyn y Christian, se enfrentan por culpa de la seducción de una espectacular mujer. Sweyn, el gemelo hercúleo de perfectas facciones y proporciones, queda enamorado de Piel Blanca, la mujer lobo, nada más verla. Christian, el gemelo menos musculoso y bello conoce la verdad e intenta advertir a su hermano acerca del peligro que corre. La narración terminará con la muerte de Christian y Piel blanca. Una vez más, el cuento comienza en un espacio doméstico destinado al confort familiar: "El gran salón de la granja estaba iluminado por la luz del fuego, y había ruido por la risa, la charla y los que estaban trabajando" (Housman, 1890: 250). Este lugar calmo no tardará en tornarse extraño a medida que la presencia de la mujer loba se acerca: "La negra boca de la chimenea, sobre el hogar, engullía, como en un misterioso remolino, espesas columnas de humo (...) y más allá, en la oscuridad, había murmullos y gemidos, así que a veces el humo se echaba atrás por el pánico" (Housman, 1890: 250). Tras la llegada de Piel blanca a la casa suceden una serie de desapariciones que inquietan a los familiares y llenan el hogar de horrores. Gracias al narrador omnisciente, el lector es testigo de la verdadera criatura que es Piel Blanca. En un principio, aparece como una mujer llamativamente bella y fuerte: "Una mujer con una capa blanca entró (...) Era una doncella, alta y muy hermosa. Sus ropas eran extrañas, medio masculinas, pero no poco femeninas" (Housman, 1890: 254). Sin embargo, la hermosa Piel Blanca tiene su lado oscuro y salvaje que palpita bajo la forma de la loba: "La terrible Cosa que estaba entre ellos, oculta por la belleza femenina, era el centro de interés" (Housman, 1890: 270).

La mujer lobo representa esa Sombra que ha de ser asumida y recuperada; deja entrever la resurrección de un poder femenino dormido y avasallado por una cultura masculina. Se representa en ella el aspecto salvaje e indestructible de la mujer, todo aquello que jamás podrá aceptar los rigores y las exigencias de una cultura muerta o excesivamente civilizadora. Como Clarissa Pinkola Estés aclara, en nuestro interior tenemos la capacidad de volver a configurarnos como la criatura salvaje que fuimos, en el interior de la mujer humana, vive también el yo instintivo y animal (Estés, 1998: 
348). En Piel Blanca se proyectan los contenidos reprimidos destinados a conformar el arquetipo. La autora, al igual que la psicoanalista Clarissa Pinkola Estés, invita a recuperar a la mujer fiera y salvaje que existió, nos invitan a integrar nuestra propia Sombra:
"Todos sentimos el anhelo de lo salvaje. Y este anhelo tiene muy pocos antídotos culturalmente aceptados. Nos han enseñado a avergonzarnos de este deseo (...) pero la Sombra de la Mujer Salvaje acecha todavía a nuestra espalda de día y de noche. Dondequiera que estemos, la Sombra que trota detrás de nosotras tiene sin duda cuatro patas" (Estés, 1998: 2).

La narradora se descubre como una clara defensora de la recuperación de ese lado dormido. Esta idea queda clara al final del relato cuando denigra al hombre que ha acabado con su vida: "La verdadera realidad era que el cerebro del hombre se encogió, se encogió hasta que se quedó en nada" (Housman, 1890: 284).

\section{CONCLUSIÓN}

La Sombra recoge los contenidos de la psique que a través de la vida no se admiten y se arrojan o reprimen. En su mayor parte la Sombra se compone de deseos reprimidos, impulsos y pasiones que se excluyen de la propia imagen individual. Mediante el análisis de este arquetipo en dos relatos pertenecientes a una época concreta y escritos por mujeres, queda expuesto que una gran parte de lo que la mujer ocultaba y reprimía no era porque ella no quisiera aceptarlo, sino porque la sociedad se lo imponía. El patriarcado dictaba lo que la mujer debía recluir en la Sombra.

El primer relato pone de manifiesto cómo la mujer debía reprimir sus aspiraciones intelectuales y sus ansias de emanciparse. La sociedad patriarcal no respetaba sus necesidades de expresarse en voz alta. La protagonista del relato se siente amordazada e impotente y guarda en su Sombra todos sus deseos inconfesables. Sin embargo, esos anhelos no pueden contenerse mucho tiempo y, tarde o temprano, salen a la luz. La Sombra es la mujer empapelada de la pared que acaba resurgiendo de las tinieblas para recordar que por más aterrador que resulte el encuentro con la Sombra es indispensable hacerle frente para iniciar el proceso de libertad. Por muy temido y difícil que sea este enfrentamiento resulta más enriquecedor que espeluznante.

En el segundo relato se refleja la Sombra del inconsciente colectivo, el temor al poder salvaje de la mujer en una sociedad manejada por los hombre. La autora defiende que no neguemos lo que la sociedad teme de nosotras y que hemos de integrarlo en nuestro individuo para convertirnos en seres más completos. Este cuento pone de manifiesto aquello que la mujer no puede ocultar por naturaleza, por mucho 
que la sociedad patriarcal quiera ocultarlo en la Sombra. La mujer lobo es en realidad una vía de escape para reflejar la seducción y la sexualidad que no podían ser expresadas abiertamente. Es un canal de desahogo para la parte instintiva negada por la sociedad.

Ambos relatos son testimonios de una época en la que la mujer comenzaba a utilizar la pluma como arma. La mujeres habían guardado durante mucho tiempo sus ansias $\mathrm{y}$, cansadas de esa visión errónea del mundo, su literatura comenzaba a esconder una fuerte crítica social. Penetrar en la estructura subjetiva de lo femenino, a través de la visión de estas autoras, es una experiencia fascinante y conmovedora.

\section{BIBLIOGRAFÍA}

AMSTRONG, N. (1987): Desire and Domestic Fiction: A Political History of the Novel, New York, Oxford University Press. Versión española traducida por María Coy (1991), Madrid, Ediciones Cátedra S.A.

BALDICK, C. (1992): The Oxford Book of Gothic Tales, New York, Oxford University Press.

BALLESTEROS, A. (2008): "El laberinto femenino de la soledad y la fantasía: cuatro escritoras victorianas frente a lo sobrenatural", Sites of Female Terror. En torno a la mujer y el terror. Estudios de mujeres, VI, 41-64.

CEPLAIR, L. (1991): Charlotte Perkins Gilman: A Non Fiction Reader, New York, Columbia University Press.

CLARK, A. (2010): Deseo. Una historia de la sexualidad en Europa, Madrid, Ediciones Cátedra, Colección Feminismos.

ESTÉS, C. P. (1998): Mujeres que corren con los lobos, Madrid, Punto de lectura.

FONDERBRIDER, J. (2005): Licantropía. Historias de Hombres Lobo en Occidente, Buenos Aires, Adriana Hidalgo Editora.

GILBERT, S. \& GUBAR, S. (1979): The Madwoman in the Attic. A Study of the Literary Imagination in the Nineteenth Century, New Haven, Yale University Press. Versión española traducida por MARTíNEZ GIMENO, C. (1998), Madrid, Ediciones Cátedra S.A.

GILMAN, C. P. (1892): "El empapelado amarillo" en NAVARRO A. J. (ed.) Venus en las tinieblas. Relatos de horror escritos por mujeres, Madrid, Valdemar gótica, 131-150.

GONZÁLEZ GARCÍA, P. D. (2009): Manifestaciones culturales del arquetipo sombra, Fundación C.G. Jung de Psicología Analítica, http://www.fundacionjung.com.ar

HOUSMAN, C. (1890): "La mujer lobo", en NAVARRO A. J. (ed.) Venus en las 
tinieblas. Relatos de horror escritos por mujeres, Madrid, Valdemar gótica, 247290.

JUNG, C. G. (1981): Recuerdos, Sueños, Pensamientos, Barcelona, Seix Barral. (2005): Símbolos de transformación, Barcelona, Paidós.

(2007): Dos escritos sobre psicología analítica, Obras completas VII, Madrid, Trotta. LAS HERAS, A. (2008): Manual de psicología junguiana, Argentina, Trama.

MARDER, H. (2000): Virginia Woolf. La medida de la vida, Buenos Aires, Adriana Hidalgo Editora.

MOERS, E. (1963): Literary Women, London, The Women's Press Limited.

RAMSLAND, K. (1994): Prism of the Night. A Biography of Anne Rice, New York, Plume.

ROBERTSON, R. (1998): Arquetipos junguianos, España, Paidós.

SHOWALTER, E. (1985): New Feminist Criticism: Essays on Women, Literature, and Theory, New York, Pantheon Books.

WOOLF, V. (1929): A Room of One's Own, London, The Hogarth Press. Versión española de PUJOL, L. (1989), Barcelona, Seix Barral. 